\title{
Appendix: Interviews
}

All 26 interviews were conducted and transcribed between 2008 and 2017. From this wider sample a core sample of ten interviews (sample A) was then selected for in-depth analysis. Note that pseudonyms are used in sample A interviews.

\section{Project interviews sample A (core sample)}

\section{Clare Cullen}

Born: 1940, grew up in north Cork

Parents: Mother, housewife; Father, sergeant in Civic Guards

Education: National School until 14, secretarial college

Migration: London 1957, Kent 1960, Cork 196?, Oldham 197 ?

Post-migration work: Bank

Marriage: 1959 , three children

Interview: 04/03/09

Reference: Clare Cullen (b. Cork, 1940) PI/SA/1

\section{Kate Daly}

Born: 1941, grew up Co. Sligo

Parents: Mother, housewife; Father, farmer and quarry owner

Education: National School until 14, convent in Roscommon

Migration: Dublin 1959, Manchester 1961

Pre-migration work: Children's nurse

Post-migration work: General nurse, factory line operative, care worker Marriage: 1964, four children 
Interview: 25/03/12

Reference: Kate Daly (b. Sligo, 1941) PI/SA/2

\section{Joe Doherty}

Born: 1944, grew up Co. Longford

Parents: Mother, housewife; Father, farmer and thatcher

Education: National School until 14, then local vocational school until 16.

Migration: Birmingham 1962, Manchester 1964

Pre-migration work: Turf cutting

Post-migration work: Factory operative, construction industry

Marriage: 1969 , four children

Interview: 27/04/08

Reference: Joe Doherty (b. Longford, 1944) PI/SA/3

\section{Bill Duffy}

Born: 1951, grew up Co. Roscommon

Parents: Mother, housewife; Father, farmer

Education: National School until 13

Migration: Manchester 1967

Post-migration work: Construction industry (labourer, site manager, executive)

Marriage: 1975 , three children

Interview: 13/03/12

Reference: Bill Duffy (b. Roscommon, 1951) PI/SA/4

\section{Brenda Grady}

Born: 1927, grew up Co. Galway

Parents: Mother, housewife; Father, farmer

Education: National School until 14

Migration: Lymme1946, Manchester 1947

Pre-migration work: Domestic servant

Post-migration work: Domestic servant, receptionist, housewife, dinner-lady

Marriage: 1949 , six children

Interview: 14/04/08

Reference: Brenda Grady (b. Galway, 1927) PI/SA/5

\section{Sean Hagan}

Born: 1935, grew up Co. Offaly 


\section{Appendix}

Parents: Mother, housewife; Father, rural labourer

Education: National School until 12

Migration: Bolton 1956

Pre-migration work: Turf cutting, beet picking, road worker, Irish army Post-migration work: Cotton mill (warehouse), construction (labourer), night watchman

Marriage: 1957 , six children

Interview: 18/02/09

Reference: Sean Hagan (b. Dublin, 1935) PI/SA/6

\section{Denis Heaney}

Born: 1927, grew up Co. Kerry

Parents: Mother, housewife; Father, farmer

Education: National School until 14

Migration: London 1948, various locations in the south-east 19481956, Leigh 1956, Stockport 1958, Manchester 1993

Pre-migration work: Turf cutting

Post-migration work: Foundry, construction (labourer, subcontractor)

Marriage: 1952, two children

Interview: 20/03/10

Reference: Denis Heaney (b. Kerry, 1927) PI/SA/7

\section{Rosie Long}

Born: 1938, grew up in Cork City

Parents: Mother, orderly at mental asylum, orderly at school for the blind; Father, left family

Education: Girls convent until 14

Migration: London 1954, Oldham 1966

Pre-migration work: Hospital orderly, orderly at school for the blind Post-migration work: Domestic servant, food server, care worker

Marriage: 1960, two children, plus four children from a second relationship

Interview: 11/02/09

Reference: Rosie Long (b. Cork, 1938) PI/SA/8

\section{Paul Quinn}

Born: 1938, grew up in Galway

Parents: Mother, housewife; Father, farmer

Education: National School until 13

Migration: Manchester 1962, London 1970

Pre-migration work: Farm labourer, building 
Post-migration work: Construction industry

Marriage: 1975, three children

Interview: $27 / 05 / 13$

Reference: Paul Quinn (b. Galway, 1938) PI/SA/9

\section{Aileen Walsh}

Born: 1927, grew up in Mayo

Parents: Mother, housewife; Father, farmer

Education: National School until 14

Migration: Doncaster 1945, Bradford 1947, Manchester 1949

Pre-migration work: Domestic

Post-migration work: Hotel maid, factory operative, waitress

Marriage: 1950, three children

Interview: 20/7/2012

Reference: Aileen Walsh (b. Mayo, 1929) PI/SA/10

\section{Project interviews sample B (wider sample)}

James Devlin (b. Kerry, 1940), interview 02/02/2010, PI/SB/1 Tony McCabe (b. Cork, 1938), interview 22/03/2009, PI/SB/2 Terry Langan (b. Longford, 1938), interview 04/05/2014, PI/SB/3 Mary Canavan (b. Sligo, 1941), interview 12/02/2013, PI/SB/4 Joseph Rooney (b. Dublin, 1950), interview 25/07/2015, PI/SB/5 Anna Kelly (b. Kerry, 1941), interview 23/07/2015, PI/SB/6 Bridget Mulvey (b. Mayo, 1939), interview 14/05/2014, PI/SB/7 Michael Walsh (b. Clare, 1946), interview 02/09/2010, PI/SB/8 Joan Kernan (b. Roscommon, 1950), interview 07/08/2014, PI/SB/9 Patrick Kernan (b. Roscommon, 1948), interview 07/08/2014, PI/SB/10 Mary McGovern (b. Sligo, 1941), interview 04/03/2014, PI/SB/11 Maeve Maher (b. Leitrim, 1938), interview 07/06/2010, PI/SB/12 Rachel O'Hare (b. Limerick, 1940), interview 09/06/2010, PI/SB/13 Donal Murtagh (b. Kerry, 1946), interview 03/11/2015, PI/SB/14 Patrick O’Dowd (b. Galway, 1941), interview 02/02/2010, PI/SB/15 Patricia Foley (b. Roscommon, 1929), interview 15/10/2009, PI/SB/16 